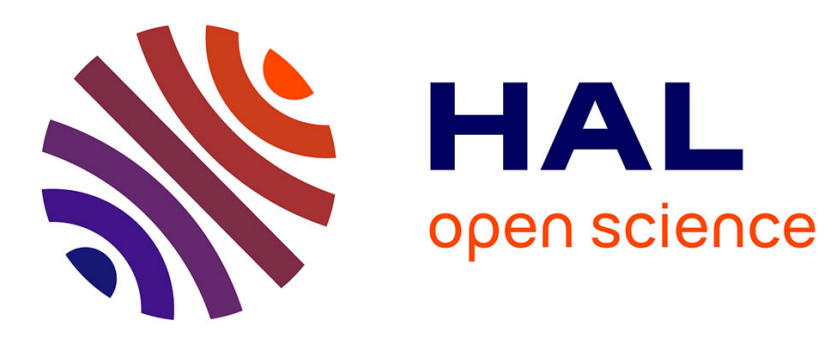

\title{
Interaction Sheaves on Continuous Domains
}

\author{
Joseph M. Abdou, Hans Keiding
}

\section{To cite this version:}

Joseph M. Abdou, Hans Keiding. Interaction Sheaves on Continuous Domains. Journal of Mathematical Economics, 2009, 45 (11), pp.708-719. 10.1016/j.jmateco.2009.05.005 . halshs-00633578

\section{HAL Id: halshs-00633578 \\ https://shs.hal.science/halshs-00633578}

Submitted on 18 Oct 2011

HAL is a multi-disciplinary open access archive for the deposit and dissemination of scientific research documents, whether they are published or not. The documents may come from teaching and research institutions in France or abroad, or from public or private research centers.
L'archive ouverte pluridisciplinaire HAL, est destinée au dépôt et à la diffusion de documents scientifiques de niveau recherche, publiés ou non, émanant des établissements d'enseignement et de recherche français ou étrangers, des laboratoires publics ou privés. 


\title{
Interaction Sheaves on Continuous Domains
}

\author{
J. Abdou* Hans Keiding ${ }^{\dagger}$ \\ Revised version \\ March 2009
}

\begin{abstract}
We introduce a description of the power structure which is inherent in a strategic game form using the concept of an interaction sheaf. The latter assigns to each open set of outcomes a set of interaction arrays, specifying the changes that coalitions can make if outcome belongs to this open set. The interaction sheaf generalizes the notion of effectivity functions which has been widely used in implementation theory, taking into consideration that changes in outcome may be sustained not only by single coalitions but possibly by several coalitions, depending on the underlying strategy choices. Also, it allows us to consider game forms with not necessarily finite sets of outcomes, generalizing the results on solvability of game forms obtained in the finite case in Abdou and Keiding (2003).
\end{abstract}

Keywords: Nash equilibrium, strong equilibrium, solvability, effectivity, acyclicity.

JEL Classification: C70, D71

AMS Classification: 91A44

*University Paris 1, CNRS, Paris School of Economics.Centre d'Economie de Paris, 106-112 Boulevard de l'Hôpital 75647 Paris Cedex 13; email: abdou@univ-paris1.fr

${ }^{\dagger}$ University of Copenhagen, Department of Economics, Studiestraede 6 DK-1455 Copenhagen K, Denmark; e-mail: Hans.Keiding@econ.ku.dk 


\section{Introduction}

A game form is strongly solvable if for each assignment of individual preferences over outcomes, the resulting game possesses a strong Nash equilibrium. Several necessary conditions for strong solvability can be found in the literature; Abdou and Keiding (2003) provide conditions which are both necesssary and sufficient, at least for the case where the strategy sets of the game form are all finite. In this paper, we extend the results obtained for finite games to the more general case where strategy sets and outcome spaces are compact Hausdorff spaces. The need for such an extension is rather obvious, since the use of mixed strategies will turn a finite game into a game with infinitely many strategies. To perform this extension, we present a model of interaction based on power distribution among agents, a model general enough to allow for a representation of classical coalitional models (e.g. effectivity functions) as well as to capture the essential features of strategic models (e.g. game forms). For this, we use an extension of the well-known concept of an effectivity function associated with a game form introduced by Moulin and Peleg (1982) and the property of acyclicity of this extended effectivity function, also known from the implementation literature, cf. e.g. Abdou and Keiding (1991). This extension is done in such a way that the essential interaction inherent in a strategic game form can be represented in the new object. An interaction sheaf is to the notion of equilibrium (e.g. Nash or strong Nash) precisely what the effectivity function is to the core.

A game form is said to be $\mathcal{M}$-solvable (where $\mathcal{M}$ is any collection of coalitions) if it has $\mathcal{M}$-equilibria for any assignment of preferences. Nash solvability and strong Nash solvability are special cases of $\mathcal{M}$-solvability. In the present case of infinite strategy spaces, the notion of an outcome-dependent effectivity function, which is at the basis of the characterization of solvability, is naturally formalized using the concept of a sheaf, which captures the idea of local (outcome-dependent) power of coalitions by specifying the power structure valid at each open set of outcomes. It turns out that the right concept in this setting is the interaction sheaf associated with a game form. Furthermore the notion of an abstract interaction sheaf is introduced and the corresponding notion of settlement set and stability.

An interaction sheaf is an object which is similar to that of an effectivity structure as introduced by Abdou and Keiding (2003), with the difference that it can be used in the context of outcome spaces that are not necessarily finite. The advantages of the present way of formalizing power structures are that (1) it allows for the representation of various equilibrium concepts within the same interaction form, whereas the effectivity structure is specific to one equilibrium concept, (2) in the current model, it allows for operations like projections that faithfully reflect the change in the underlying coalition structure, and, most importantly, (3) since only the interactive form associated to some game form and some equilibrium concept is relevant for stability, it allows for a simple comparison between different 
procedures or mechanisms with respect to stability. An interaction form can thus be viewed as an intrinsic representation of power without a direct reference to strategies or to some equilibrium concept.

The paper is structured as follows: In Section 2, we give the definitions of the basic concepts such as game forms, preferences, equilibria, and in Section 3, we proceed to the concepts which are central for the following, namely interaction sheaves. In this section, we also investigate some general properties of interaction sheaves, and we introduce the notion of a settlement as well as stability of interaction sheaves. The next section is concerned with the characterization of stable interaction sheaves by the property of acyclicity, thus extending a result known from simple games (Nakamura (1979)) and effectivity functions (Keiding (1985)) to the present general context of interaction sheaves. Section 5 contains the main result of the paper, showing that solvability of game forms may be characterized in terms of stability or acyclicity of the associated interaction sheaf, and Section 6 gives a brief treatment of the special case where the set of alternatives is convex, and where there are also convexity conditions on individual preferences. The concluding section contains some final comments.

\section{Basic definitions and notations}

In the present section, we introduce the concepts and the notation which will be needed as we proceed. We use the following notational conventions: For any set $D$, we denote by $\mathcal{P}(D)$ the set of all subsets of $D$ and by $\mathcal{P}_{0}(D)=\mathcal{P}(D) \backslash\{\emptyset\}$ the set of all nonempty subsets of $D$. Elements of $\mathcal{P}(N)$ are called coalitions; an active coalition structure (shorthand ACS) is a subset $\mathcal{M}$ of $\mathcal{P}_{0}(N)$.

A game form is an array $G=\left(X_{1}, \cdots, X_{n}, A, g\right)$. Here the set $N=\{1, \cdots, n\}$, where $n \geq 2$, is interpreted as the set of players, $X_{i}$ is the strategy set of player $i$, for $i \in N, A$ is the set of alternatives, and $g: \prod_{i \in N} X_{i} \longrightarrow A$ is the outcome function. For every coalition $S \in \mathcal{P}_{0}(N)$, the product $\prod_{i \in S} X_{i}$ is denoted $X_{S}$ (by convention $X_{\emptyset}$ is the singleton $\{\emptyset\}$ ) and $N \backslash S$ is denoted $S^{c}$. Similarly if $B \in \mathcal{P}(A), A \backslash B$ is denoted $B^{c}$. If $x_{N} \in X_{N}$, the notation $g\left(x_{S}, X_{S^{c}}\right)$ stands for $\left\{g\left(x_{S}, y_{S^{c}}\right) \mid y_{S^{c}} \in X_{S^{c}}\right\}$ if $S \neq \emptyset$ and for $g\left(X_{N}\right)$ if $S=\emptyset$.

For any set $D, Q(D)$ denotes the set of all quasi-orders on $D$ (that is all binary

relations on $D$ which are complete and transitive). We let $\stackrel{\circ}{R}$ denote the strict preference relation associated with $R$, that is $a \stackrel{\circ}{R} b$ if and only if $a R b$ and not $b R a$.

We assume that $X_{1}, \ldots, X_{n}$ and $A$ are Hausdorff compact topological spaces and that $g$ is continuous and onto. We denote by $\mathcal{G}$ the set of open sets of $A$, by $\mathcal{F}$ that of closed sets. Moreover $\mathcal{G}_{0} \equiv \mathcal{G} \backslash\{\emptyset\}$ and $\mathcal{F}_{0} \equiv \mathcal{F} \backslash\{\emptyset\}$. A quasi-order $R$ on $A$ is continuous if for any $a \in A$ the sets $\{b \in A \mid b R a\}$ and $\{b \in A \mid a R b\}$ are closed. A continuous real function $u$ induces a continuous quasi-order $R$ by 
setting $a R b$ if and only if $u(a) \geq u(b)$.

A game in strategic form is an array $\left(X_{1}, \ldots, X_{n} ; Q_{1}, \ldots, Q_{n}\right)$, where for each $i \in N, X_{i}$ is the set of strategies of player $i$, and $Q_{i}$ is a quasi-order on $X_{N}=$ $\prod_{i \in N} X_{i}$. For $\mathcal{M}$ an ACS (active coalition structure), a strategy array $x_{N} \in X_{N}$ is an $\mathcal{M}$-equilibrium of the game $\left(X_{1}, \ldots, X_{n} ; Q_{1}, \ldots, Q_{n}\right)$ if there is no coalition $S \in \mathcal{M}$ and $y_{S} \in X_{S}$ such that for all $i \in S$ :

$$
\left(y_{S}, x_{S^{c}}\right) \stackrel{\circ}{Q_{i}} x_{N}
$$

For $R \in Q(A)$ we put $P(a, R)=\{b \in A \mid b \stackrel{\circ}{R} a\}$. A preference profile (over $A)$ is a map $R_{N}$ from $N$ to $Q(A)$, also written as $R_{N}=\left(R_{1}, \ldots, R_{n}\right)$, so that a preference profile is an element of $Q(A)^{N}$. For notational convention, we write $P\left(a, i, R_{N}\right)$ for $P\left(a, R_{i}\right)$, and we use the notations $P\left(a, S, R_{N}\right)=\cap_{i \in S} P\left(a, i, R_{N}\right)$ for $S \in \mathcal{P}_{0}(N)$. For each preference profile $R_{N} \in Q(A)$, the game form $G$ induces a game $\left(X_{1}, \ldots, X_{n} ; Q_{1}, \ldots, Q_{n}\right)$ with the same strategy spaces as in $G$ and with the $Q_{i}$ defined by

$$
x_{N} Q_{i} y_{N} \Leftrightarrow g\left(x_{N}\right) R_{i} g\left(y_{N}\right)
$$

for $x_{N}, y_{N} \in X_{N}$. We denote this game by $\left(G, R_{N}\right)$

We say that $a \in A$ is an $\mathcal{M}$-equilibrium outcome of $\left(G, R_{N}\right)$ if there exists an $\mathcal{M}$-equilibrium $x_{N}$ in $\left(G, R_{N}\right)$ such that $g\left(x_{N}\right)=a$. Let $L \subset Q(A)$ be a subset of preferences. The game form $G$ is said to be solvable in $\mathcal{M}$-equilibrium or $\mathcal{M}$-solvable on $L$ if for each preference profile $R_{N} \in L^{N}$, the game $\left(G, R_{N}\right)$ has an $\mathcal{M}$-equilibrium. In particular, when $\mathcal{M}=\mathcal{N}=\{\{1\}, \ldots,\{n\}\}$, the set of all singleton coalitions, then an $\mathcal{M}$-equilibrium is simply a Nash equilibrium. Similarly, when $\mathcal{M}=\mathcal{P}_{0}(N)$, the family of all coalitions, an $\mathcal{M}$-equilibrium is a strong Nash equilibrium.

\section{Interaction sheaves}

In this paper, we aim at a characterization of $\mathcal{M}$-solvable game forms using a suitable notion of power structure which is inherent in the game form. This approach was initiated by the seminal paper by Moulin and Peleg (1984), where they introduced the effectivity function associated with a game form. For the solvability of game forms, the effectivity function contains too little information, and refined notions of power structures were considered by Abdou and Keiding (2003) in the context of solvability of game forms with finite strategy spaces. Below we introduce a generalization of effectivity functions that will work in the context of solvability of game forms with an infinite number of strategies.

The basic extension of the concept of an effectivity function needed in order to treat solvability problems consists in changing the focus from coalitions taking 
joint action, which is the scenario considered in simple games or effectivity functions, to collections of coalitions acting independently but simultaneously. The formalization has to take this into account, and this leads to the concept of an interaction array to be defined below as an instance of simultanous coalitional action. In the interpretation, where one associates this extended concept to a game form, these collections of coalitions acting simultaneously arise in a natural way: an outcome can be overruled if no matter by which strategy array it was achieved there is one of the coalitions in the collection which can force outcome to be something else.

Definition 3.1 (a) An interaction array on $(N, A)$ is a map $\varphi: \mathcal{P}_{0}(N) \rightarrow \mathcal{P}(A)$ with $\varphi(S) \neq \emptyset$ for some $S \in \mathcal{P}_{0}(N)$. Let $\mathcal{P}_{0}(N, A)$ be the set of all interaction arrays. We introduce a partial order $\leq$ on $\mathcal{P}_{0}(N, A)$ by the formula $\varphi \leq \psi$ if and only if $\varphi(S) \subset \psi(S)$ for all $S \in \mathcal{P}_{0}(N)$. For $\mathcal{A} \subset \mathcal{P}(A)$. We denote by $\mathcal{A}_{0}(N, A)$ the set of all interaction arrays with values in $\mathcal{A}$.

(b) An interaction form with values in $\mathcal{A}$ is a map $\mathcal{E}: \mathcal{G}_{0} \rightarrow \mathcal{P}\left(\mathcal{A}_{0}(N, A)\right)$ such that for all $U \in \mathcal{G}_{0}, \varphi, \psi \in \mathcal{A}_{0}(N, A)$, if $\varphi \in \mathcal{E}[U]$ and $\varphi \leq \psi$, then $\psi \in \mathcal{E}[U]$.

(d) The interaction form $\mathcal{E}$ is a an interaction presheaf if $U \subset V \Rightarrow \mathcal{E}[V] \subset \mathcal{E}[U]$ for all $U, V \in \mathcal{G}_{0}$, and an interaction sheaf if, in addition, for each $U \in \mathcal{G}_{0}$ and each open covering $\left(U_{i}\right)_{i \in I}$ of $U$ one has $\mathcal{E}[U]=\cap_{i \in I} \mathcal{E}\left[U_{i}\right]$.

Interaction forms are collections of interaction arrays that satisfy natural conditions of non-trivialness and monotonicity, and they can be considered as a formalization of the power structure in society. Part (c) of the definition connects the power structure of the interaction form to the topology of the outcome space.

The notion of an interaction presheaf extends that of an effectivity function in two distinct ways. Apart from the shift of focus from coalitions to collections of coalitions, which was mentioned above, the interaction presheaf incorporates a local approach to coalitional power, in the sense that a collection of coalitions may be effective for upsetting some choices by society but not other choices. This local aspect which makes it natural to use the notion of a sheaf in the formalization of this power structure.

By a slight abuse of language, we say that an interaction presheaf $\mathcal{E}$ is closedvalued (open-valued) if for all $U \in \mathcal{G}_{0}$, each $\varphi \in \mathcal{E}[U]$ belongs to $\mathcal{F}_{0}(N, A)$ $\left(\mathcal{G}_{0}(N, A)\right)$, so that not the set $\mathcal{E}[U]$ but all the intersection arrays in this set have closed (open) values.

When $\mathcal{E}$ is a presheaf, we may think of an interaction array in $\mathcal{E}[U]$ as a description of an available move of the agents given any state in $U$. In order that a scenario leading to some outcome be viable, it needs to be approved by all coalitions. To interpret the statement $\varphi \in \mathcal{E}[U]$, one may imagine that any outcome in $U$ can occur in different scenarios that are not directly explicited in 
the model; any scenario leading to some state in $U$ may arouse some coalition $S \in \mathcal{M}$ that objects by threatening to drive the outcome into $\varphi(S)$, and in this case $a$ is rejected. Implicitly, $a$ may arise in many different scenarios. In order to disrupt $a$ the simultaneous move of all coalitions may be needed; these moves are interpreted as being disjunctive and simultaneous. When a coalition structure becomes active at $a$, this activation must be understood as a collusion of interests between its components. Indeed the rejection of $a$ is equivalent to the rejection of each scenario leading to $a$, and each scenario may be opposed by some coalition in $\mathcal{M}$. Our model is universal in the sense that we allow a priori all coalitions to react to some state in $U$. Nevertheless, the fact that $\varphi(S)=\emptyset$ for some $S$ means that coalition $S$ is inhibited or desactivated and therefore that the power represented by $\varphi$ holds without the participation of $S$. Therefore the support of $\varphi$ (i.e. those coalitions $S$ for which $\varphi(S)$ is nonempty) is in fact the active coalition structure behind $\varphi$.

Remark 3.2 The discussion of the present section has been confined to situations where the coalition structure is $\mathcal{P}_{0}(N)$, the set of all nonempty subsets of $N$. However, restricting to any $\mathcal{M} \subset \mathcal{P}_{0}(N)$ means simply that we consider only interaction arrays which are projections $\left.\varphi\right|_{\mathcal{M}}$ to $\mathcal{M}$ of interaction arrays $\varphi \in \mathcal{P}_{0}(N, A)$, where

$$
\begin{gathered}
\left.\varphi\right|_{\mathcal{M}}(S)=\left\{\begin{array}{l}
\varphi(S) \text { if } S \in \mathcal{M}, \\
\emptyset \text { otherwise. }
\end{array}\right. \\
\left.\mathcal{E}\right|_{\mathcal{M}}[U]=\left\{\varphi \in \mathcal{A}_{0}(N, A)|\varphi|_{\mathcal{M}}(S) \in \mathcal{E}[U]\right\}
\end{gathered}
$$

It turns out (see Proposition 5.2 and Remark 5.1) that this restriction reflects faithfully what is meant when the coalitions that are allowed to act jointly are the members of the ACS $\mathcal{M}$.

As a first example of an interaction form, we consider the one induced by an effectivity function $E$, that is a map $E: \mathcal{P}_{0}(N) \rightarrow \mathcal{P}\left(\mathcal{P}_{0}(A)\right)$. To define the interaction form $\mathcal{E}^{E}$ associated with $E$ we let $\mathcal{E}^{E}[U]$, for $U \in \mathcal{G}_{0}$, contain all the interaction arrays $\varphi$ such that $\delta_{(S, B)} \leq \varphi$, for $B \in E(S)$, where

$$
\delta_{(S, B)}\left(S^{\prime}\right)=\left\{\begin{array}{l}
B \text { if } S^{\prime}=S, \\
\emptyset \text { otherwise. }
\end{array}\right.
$$

This is an interaction sheaf, which is constant in the sense that $\mathcal{E}^{E}[U]$ does not depend on $U$. Similarly, given an interaction form $\mathcal{E}$ one can extract an effectivity functions $E^{\mathcal{E}}$ with

$$
E^{\mathcal{E}}(S)=\left\{B \in P_{0}(A) \mid \delta(S, B) \in \mathcal{E}[A]\right\}
$$


For a more interesting interaction form, consider a game form $G=\left(X_{1}, \ldots, X_{n}\right.$, $A, g)$ and $\mathcal{M} \subset \mathcal{P}_{0}(N)$. The $\beta$-interaction form associated with $(G, \mathcal{M})$ is defined as

$$
\begin{aligned}
\mathcal{E}_{\beta}^{G, \mathcal{M}}[U]=\left\{\varphi \in \mathcal{P}_{0}(N, A) \quad \mid\right. & \forall x_{N} \in g^{-1}(U), \\
& \left.\exists S \in \mathcal{M}, \exists y_{S} \in X_{S}: g\left(y_{S}, x_{S^{c}}\right) \in \varphi(S)\right\}
\end{aligned}
$$

for all $U \in \mathcal{G}_{0}$. Thus, the $\beta$-interaction presheaf associated with $G$ contains at the open set $U$ all the interaction forms $\varphi$ which are such that any strategy array with outcome in $U$ can be modified by at least one coalition $S$ so as to have its outcome in $\varphi(S)$. This is actually an interaction sheaf. As is usual, the $\beta$-construction shows what coalitions can do by adapting their coordinated strategy choices to the situation, the latter represented by a strategy array with outcome in $U$. There is a corresponding $\alpha$-construction: Define the $\alpha$-interaction form associated with $G$ and $\mathcal{M}$ by

$$
\begin{aligned}
\mathcal{E}_{\alpha}^{G, \mathcal{M}}[U]=\left\{\varphi \in \mathcal{P}_{0}(N, A) \mid\right. & \exists x_{N} \in g^{-1}(U), \\
& \left.\forall S \in \mathcal{M}, \forall y_{S} \in X_{S}: g\left(y_{S}, x_{S^{c}}\right) \in \varphi(S)\right\} .
\end{aligned}
$$

The $\alpha$-interaction form is in general not a sheaf nor even a presheaf. It assigns to any open set the interaction arrays which specify for each coalition $S$ a set of outcomes, namely $\varphi(S)$ that $S^{c}$ can force, given some fixed strategy array. In Section 5 we study interaction forms associated with continuous game forms.

Example 3.3 Let $G$ be a game form belonging to an $n$-person unanimity game with infinite strategy spaces: Each player has strategy space $X$, where $X$ is a compact topological space, at the outcome space is $X \cup\{*\}$, where $*$ a distinguished point not belonging to $X$; the outcome function $\pi:(X \cup\{*\})^{n} \rightarrow X \cup\{*\}$ is given by

$$
\pi\left(x_{1}, \ldots, x_{n}\right)= \begin{cases}x & \text { if } x_{1}=x_{2}=\cdots=x_{n}=x \\ * & \text { otherwise }\end{cases}
$$

We want to find $\mathcal{E}_{\beta}^{G}[U]$ for some open set $U$, beginning with the case where $U \subset X$. Some of the interaction arrays in $\mathcal{E}_{\beta}^{G}[U]$ are easily identified: If $\varphi(N) \neq \emptyset$, then $\varphi \in \mathcal{E}_{\beta}^{G}[U]$, since the grand coalition can obtain any outcome in $X \cup\{*\}$. Next, every coalition with more than one member can obtain $*$ by having its members choosing different strategies, and similarly every collection of coalitions can obtain $*$ responding on any strategy array with outcome in $U$ by choosing different strategies. The corresponding interaction arrays are all $\varphi$ satisfying $* \in \varphi(S)$ for some coalition $S$.

Finally, in the game form $G$, strategies giving outcomes in $U$, that is strategies which are all equal to some $x \in U$, may be left unchanged by coalitions, meaning 
that by doing nothing they obtain that outcome belongs to $U$, The interaction forms corresponding to this option are all $\varphi$ with $U \subset \varphi(S)$ for some $S$.

Summing up, we have

$$
\mathcal{E}_{\beta}^{G}[U]=\{\varphi \mid \varphi(N) \neq \emptyset\} \cup\{\varphi \mid * \in R(\varphi)\} \cup\{\varphi \mid U \subset R(\varphi)\}
$$

where we have used the notation $R(\varphi)=\{x \in A \mid \exists S: x \in \varphi(S)\}$.

If $U$ is the set $\{*\}$, which is open in $X \cup\{*\}$, then finding all intersection arrays is somewhat more complicated, so we restrict to the case where $\mathcal{M}=$ $\{N\} \cup\{N \backslash\{i\} \mid i \in N\}$. We have as before that $\mathcal{E}_{\beta}^{G, \mathcal{M}}[U]$ contains $\{\varphi \mid \varphi(N) \neq \emptyset\}$ and $\{\varphi \mid * \in R(\varphi)\}$. To obtain something different from $*$, a coalition should be able to equalize all strategy choices. Coalitions $N \backslash\{i\}$ for $i \in N$ can always do this, and interaction arrays $\varphi$ such that $\varphi(N \backslash\{i\})=A \backslash\{a\}$ for each $i \in N$, with $R(\varphi)=A \backslash\{a\}$, will belong to $\mathcal{E}_{\beta}^{G, \mathcal{M}}[\{*\}]$. However, it is easily seen that $\mathcal{E}_{\beta}^{G, \mathcal{M}}[\{*\}]$ contains no interaction arrays $\phi$ for which $R(\varphi)$ is strictly smaller than $A \backslash a$ for some $a \in A$.

Whether coalitions in the ACS have a real interest to dismantle an outcome $a$, depends on the actual preferences. This is why we introduce the following:

Definition 3.4 Let $\mathcal{E}$ be an interaction presheaf, and let $R_{N} \in Q(A)^{N}$ be a preference profile. The alternative $a \in A$ is dominated in $\mathcal{E}$ at $R_{N}$ if there is an open neighbourhood $U$ of $a$ and an interaction array $\varphi \in \mathcal{E}[U]$ such that for all $S \in \mathcal{P}_{0}(N), \varphi(S) \subset P\left(a, S, R_{N}\right)$.

A settlement (for $\mathcal{E}$ at $R_{N}$ ) is an alternative which is not dominated at $R_{N}$; the set of all settlements is denoted $\operatorname{Stl}\left(\mathcal{E}, R_{N}\right)$. For $L$, a set of preferences, the interaction presheaf $\mathcal{E}$ is stable on $L$ if $\operatorname{Stl}\left(\mathcal{E}, R_{N}\right)$ is nonempty for all profiles $R_{N} \in L^{N}$.

The definition of domination given here corresponds to the usual one, given that the fundamental instrument of domination, which in the context of simple games or effectivity functions was a coalition and an alternative or a subset of alternatives, has been replaced by the interaction array as expressing the collection of coalition-subset pairs that may block a particular outcome. The set of undominated alternatives, which corresponds to the standard notion of the core, is called the settlement set to prevent possible confusion.

An outcome $a$ is a settlement if there exists at least one scenario that forces $a$ and is such that no active coalition has an incentive to disrupt it. Thus, the notion of a settlement generalizes the well-known concept of the core; since the notion of domination is more general, going beyond what is achieved by a single coalition working separately, we have chosen to use another term for the set of undominated alternatives. 
In the next section, we shall consider combinatorial conditions on the interaction presheaf $\mathcal{E}$ which implies that it is stable. In the remainder of this section, we shall have a closer look at the topological properties of $\mathcal{E}$.

First of all we notice that there is a quasi-order $\subset$ defined on interaction presheaves by

$$
\mathcal{E} \subset \mathcal{E}^{\prime} \text { if and only if } \mathcal{E}[U] \subset \mathcal{E}^{\prime}[U] \text { for all } U \in \mathcal{G}_{0} .
$$

Since the intersection of any family of interaction presheaves (sheaves) over $A$ is an interaction presheaf (sheaf), and since the trivial sheaf defined by $\mathcal{E}^{\prime}[U=$ $\mathcal{A}_{0}(N, A)$ for all $U \in \mathcal{G}_{0}$ contains all presheaves, it follows that for any interaction presheaf $\mathcal{E}$, there is a minimal (for $\subset$ ) interaction sheaf (called the sheaf cover of $\mathcal{E}$ and denoted $\mathcal{E}^{+}$) containing $\mathcal{E}$, whereby for any $U \in \mathcal{G}, \mathcal{E}^{+}[U]$ is defined as the intersection of all $\mathcal{E}^{\prime}[U]$ with $\mathcal{E}^{\prime} \supset \mathcal{E}$, and $\mathcal{E}^{\prime}$ is a sheaf.

Proposition 3.5 Let $\mathcal{E}$ be an interaction presheaf with values in $\mathcal{A}$, and for each open set $U$, let $\mathcal{R}(U)$ be the set of all open coverings of $U$. Then

$$
\mathcal{E}^{+}[U]=\bigcup_{R \in \mathcal{R}(U)} \bigcap_{V \in R} \mathcal{E}[V]
$$

and $\operatorname{Stl}\left(\mathcal{E}, R_{N}\right)=\operatorname{Stl}\left(\mathcal{E}^{+}, R_{N}\right)$ for any profile $R_{N}$.

Proof: Let $\mathcal{E}^{\prime}[U]$ denote the expression on the right hand of (4), then it is staightforward that $\mathcal{E}^{\prime}$ is a sheaf. Moreover for $U$ open, $\mathcal{E}[U] \subset \mathcal{E}^{\prime}[U]$ trivially, since $U$ is itself a covering of $U$, so $\mathcal{E}^{+}[U] \subset \mathcal{E}^{\prime}[U]$. It follows that $\mathcal{E}^{+} \subset \mathcal{E}^{\prime}$. Conversely if $\mathcal{E}^{\prime \prime}$ is a sheaf such that $\mathcal{E}^{\prime \prime} \supset \mathcal{E}$, then it is easy to see that $\mathcal{E}^{\prime \prime} \supset \mathcal{E}^{\prime}$. It follows that $\mathcal{E}^{+} \supset \mathcal{E}^{\prime}$. The second statement follows from the definition of domination.

Our definition of an interaction form, designed so as to capture the phenomenon of state-dependent power structure, has taken as primitive notion of local power the interaction arrays corresponding to the open neighbourhoods of the topology, corresponding to the classical notion of a presheaf (cf. eg. Bredon, 1990). Alternatively, we might have considered local power as defined for each point of $A$ (corresponding to studying the sections of a presheaf). Below, we consider such an alternative approach. In the terminology of sheaf theory, this corresponds to looking at the stalks of the sheaf at each point, which in some cases may be more appropriate.

Definition 3.6 An interaction bundle with values in $\mathcal{A}$ is a map $\mathcal{I}: A \rightarrow$ $\mathcal{A}_{0}(N, A)$. The alternative $a \in A$ is dominated in $\mathcal{I}$ at the profile $R_{N} \in Q(A)^{N}$ if there exists $\varphi \in \mathcal{I}[a]$ such that for all $S \in \mathcal{P}_{0}(N): \varphi(S) \subset P\left(a, S, R_{N}\right)$. The set $\operatorname{Stl}\left(\mathcal{I}, R_{N}\right)$ of settlements for $\mathcal{I}$ at $R_{N}$ consists of all the alternatives which are not dominated in $\mathcal{I}$ at $R_{N}$. 
It turns out that the $\beta$-interaction bundle associated with a game form is related in a straightforward manner to the question of existence of equilibria of that game form (Proposition 5.2). On the other hand the relation between acyclicity and stability is easier to express using interaction presheaves (Theorem 4.4) We now explore the interrelations between the two objects. To every interaction presheaf $\mathcal{E}$ we associate the interaction bundle $\mathcal{E}^{\bullet}$ defined by

$$
\mathcal{E}^{\bullet}[a]=\bigcup_{U \in \mathcal{U}(a)} \mathcal{E}[U]
$$

where $\mathcal{U}(a)$ is the set of all open neighbourhoods of $a$. The following proposition is obvious.

Proposition 3.7 Let $\mathcal{E}$ be an interaction presheaf. Then for any preference profile $R_{N} \in Q(A)^{N}, \operatorname{Stl}\left(\mathcal{E}, R_{N}\right)=\operatorname{Stl}\left(\mathcal{E}^{\bullet}, R_{N}\right)$.

Conversely, to any interaction bundle $\mathcal{I}$ we may associate an interaction sheaf $\mathcal{I}^{\diamond}$ defined by

$$
\mathcal{I}^{\diamond}[U]=\bigcap_{a \in U} \mathcal{I}[a]
$$

for any $U \in \mathcal{G}$. The question whether any sheaf can be obtained in this way is answered in the following:

Proposition 3.8 For any interaction presheaf $\mathcal{E}$ one has $\mathcal{E} \subset \mathcal{E}^{\bullet \diamond}$ and $\mathcal{E}^{+}=\mathcal{E}^{\bullet}$. Moreover $\mathcal{E}$ is a sheaf if and only if $\mathcal{E}=\mathcal{E}^{\bullet}$. It follows that any sheaf $\mathcal{E}$ can be obtained as $\mathcal{I}^{\diamond}$ where $\mathcal{I}$ is the interaction bundle $\mathcal{E}^{\bullet}$.

Proof: The interaction form $\mathcal{E}^{\bullet \diamond}=\left(\mathcal{E}^{\bullet}\right)^{\diamond}$ is a sheaf by its construction, and clearly $\mathcal{E} \subset \mathcal{E}^{\bullet \diamond}$, so that $\mathcal{E}^{+} \subset \mathcal{E}^{\bullet}$. Let $U \in \mathcal{G}_{0}$, let $\mathcal{E}^{\prime} \supset \mathcal{E}$ be a sheaf, and for any $a \in A$ let $\mathcal{U}(a)$ be the set of all open neighbourhoods of $a$. Writing out the definitions and using inclusion, we have that

$$
\mathcal{E}^{\bullet \diamond}[U]=\bigcap_{a \in U} \bigcup_{V \in \mathcal{U}(a)} \mathcal{E}[V] \subset \bigcap_{a \in U} \bigcup_{V \in \mathcal{U}(a)} \mathcal{E}^{\prime}[V]
$$

Now we have (set theoretic equality):

$$
\bigcap_{a \in U} \bigcup_{V \in \mathcal{U}(a)} \mathcal{E}^{\prime}[V]=\bigcup_{\left(V_{b}\right) \in \Pi_{b \in U} \mathcal{U}(b)} \bigcap_{b \in U} \mathcal{E}^{\prime}\left[V_{b}\right]
$$

where the union is over all possible collections $V=\left(V_{b}\right)_{b \in U}$, with $V_{b} \in \mathcal{U}(b)$ for all $b \in U$. Since $\mathcal{E}^{\prime}$ is a sheaf, we have that $\cap_{b \in U} \mathcal{E}^{\prime}\left[V_{b}\right]=\mathcal{E}^{\prime}\left[\cup_{b \in U} V_{b}\right] \subset \mathcal{E}^{\prime}[U]$. It follows that $\mathcal{E}^{\bullet}[U] \subset \mathcal{E}^{\prime}[U]$. Since $\mathcal{E}^{\bullet \diamond} \subset \mathcal{E}^{\prime}$ for all $\mathcal{E}^{\prime} \supset \mathcal{E}$, we have $\mathcal{E}^{\bullet \diamond} \subset \mathcal{E}^{+}$. 
Proposition 3.9 (i) For any interaction presheaf $\mathcal{E}, \mathcal{E}^{\bullet}=\left(\mathcal{E}^{+}\right)^{\bullet}$.

(ii) For presheaves $\mathcal{E}$ and $\mathcal{F}, \mathcal{E}^{\bullet}=\mathcal{F}^{\bullet}$ if and only if $\mathcal{E}^{+}=\mathcal{F}^{+}$.

Proof: (i) Let $a \in A$. Since $\mathcal{E} \subset \mathcal{E}^{+}$one has $\mathcal{E}^{\bullet}(a) \subset\left(\mathcal{E}^{+}\right)^{\bullet}(a)$. Let $\varphi \in\left(\mathcal{E}^{+}\right)^{\bullet}(a)$. then for some open neigborhood $U$ of $a, \varphi \in \mathcal{E}^{+}(U)$ and by Proposition 3.8, there exists some open covering $\left(U_{i}\right)_{i \in I}$ of $U$ such that $\varphi \in \mathcal{E}\left(U_{i}\right)$ for all $i \in I$. Since there exists $i_{0} \in I$ such that $a \in U_{i_{0}}$ we have that $\varphi \in \mathcal{E}^{\bullet}[a]$.

(ii) It follows from (i) that the equality $\mathcal{E}^{+}=\mathcal{F}^{+}$implies $\mathcal{E}^{\bullet}=\mathcal{F}^{\bullet}$. Conversely if $\mathcal{E}^{\bullet}=\mathcal{F}^{\bullet}$, then by Proposition 3.8, $\mathcal{E}^{+}=\mathcal{E}^{\bullet}=\mathcal{F}^{\bullet \diamond}=\mathcal{F}^{+}$.

We remark that an interaction bundle $\mathcal{I}$ may be not equal to $\mathcal{I}^{\bullet \bullet}$, that is for some $a \in A$, it may be the case that $\mathcal{I}(a) \neq \cup_{U \in \mathcal{U}(a)} \mathcal{I}^{\wedge}[U]$.

Example 3.10 Let $f: A \rightarrow A$ be any map and for any $a \in A$, let $\mathcal{U}(a)$ be the set open neighborhoods of $a$. Then the map $\mathcal{I}^{f}: A \rightarrow \mathcal{P}_{0}(A)$ defined by

$$
\mathcal{I}^{f}[a]=\{f(U) \mid U \in \mathcal{U}(a)\}
$$

can be viewed as an interaction bundle, since subsets of $A$ can be identified with elements of $\mathcal{P}_{0}(\{1\}, A)$. We have that

$$
\mathcal{I}^{f}[a]=\cup_{U \in \mathcal{U}(a)}\left(\mathcal{I}^{f}\right)^{\diamond}[U]
$$

if and only if $f$ is continuous at $a$.

This justifies the following:

Definition 3.11 An interaction bundle $\mathcal{I}$ is said to be regular if $\mathcal{I}=\mathcal{I}^{\triangleright \bullet}$.

If $\mathcal{I}$ is regular, then for any preference profile $R_{N} \in Q(A)^{N}, \operatorname{Stl}\left(\mathcal{I}, R_{N}\right)=$ $\operatorname{Stl}\left(\mathcal{I}^{\diamond}, R_{N}\right)$; this is a consequence of Proposition 3.7.

Proposition 3.12 Let $\mathcal{I}$ be an interaction bundle. In order that $\mathcal{I}$ be regular it is necessary and sufficient that $\mathcal{I}=\mathcal{E} \bullet$ for some presheaf $\mathcal{E}$; moreover in this case there exists a unique sheaf $\mathcal{E}$ such that $\mathcal{I}=\mathcal{E}^{\bullet}$, namely $\mathcal{E}=\mathcal{I}^{\diamond}$.

Proof. The result follows immediately from Propositions 3.8 and 3.9.

One can summarrize the situation as follows: The operation $\bullet$ takes an interaction presheaf to some regular interaction bundle, and its restriction to the set of sheaves is injective, its inverse being the operation $\diamond$. 


\section{$4 \quad$ Stability of interaction presheaves}

In this section, we introduce a combinatorial property of interaction presheaves which is shown to be equivalent to stability. This extends the results of Abdou and Keiding (2003) to the topological setup, given that the interaction presheaf satisfies a mild continuity assumption and preferences are representable by continuous real functions.

The notion of a cycle is basic in considerations of stability, that is nonemptiness of the core (of effectivity functions) or the settlement set (of interaction presheaves) for all assignments of preferences. It generalizes the ideas introduced by Nakamura (1979) in the context of simple games: If every alternative is dominated for some assignment of preferences to individuals, then there must be a configuration of dominating entities (coalition-subset pairs or interaction arrays, depending on the situation) which are mutually compatible in the sense that they can occur for a particular assignment of preferences. This latter condition constitutes the key part of the definition of a cycle; it can be restated in a way as to indicate, that for each individual the participation in the different instances of domination should be consistent with some quasi-order on the set of alternatives.

To define a cycle, we need some notation: For $\varphi \in \mathcal{P}_{0}(N, A)$ and $i \in N$ the $i$-range of $\varphi$ is the set

$$
R^{i}(\varphi)=\bigcup_{S: i \in S} \varphi(S)
$$

Definition 4.1 Let $\mathcal{E}$ be an interaction presheaf. A cycle in $\mathcal{E}$ is a family $\left(U^{k}, \varphi^{k}\right)_{k=1}^{r}$, where $U^{k} \in \mathcal{G}, \varphi^{k} \in \mathcal{E}\left[U^{k}\right], k=1, \ldots, r$ with the properties:

(i) $\cup_{k=1}^{r} U^{k}=A$,

(ii) if $i \in N$ and $\emptyset \neq J \subset\{1, \ldots, r\}$ then there exists $k \in J$ such that $U^{k} \cap R^{i}\left(\varphi^{j}\right)=\emptyset$ for all $j \in J$.

If $\mathcal{E}$ has no cycles, then $\mathcal{E}$ is said to be acyclic.

We shall also need an alternative formulation of the condition defining a cycle.

Lemma 4.2 Let $\mathcal{E}$ be an interaction presheaf. Let $\mathcal{Z}:=\left(U^{k}, \varphi^{k}\right)_{k=1}^{r}$, be a family where $U^{k} \in \mathcal{G}, \varphi^{k} \in \mathcal{E}\left[U^{k}\right], k=1, \ldots, r$ and $\cup_{k=1}^{r} U^{k}=A$, Then the following are equivalent:

(1) $\mathcal{Z}$ is a cycle of $\mathcal{E}$,

(2) For each $i \in N$ there is a permutation $\left(k_{1}, \ldots, k_{r}\right)$ of $(1, \ldots, r)$ such that for any $j \in\{1, \ldots, r\}$,

$$
\left[U^{k_{1}} \cup \cdots \cup U^{k_{j}}\right] \cap\left[R^{i}\left(\varphi^{k_{j}}\right) \cup \cdots \cup R^{i}\left(\varphi^{k_{r}}\right)\right]=\emptyset .
$$

ProOF: $(1) \Rightarrow(2)$ : Let $\left(U^{k}, \varphi^{k}\right)_{k=1}^{r}$ be a cycle in $\mathcal{E}$. We check that the family $\left(U^{k}, \varphi^{k}\right)_{k=1}^{r}$ satisfies (2). Indeed, let $i \in N$ be arbitrary. By property (ii) in 
Definition 4.1 applied to $J=\{1, \ldots, r\}$, we get the existence of $k_{1} \in\{1, \ldots, r\}$ such that $U^{k_{1}}$ and $\cup_{k=1}^{r} R^{i}\left(\varphi^{k}\right)$ have empty intersection. Now, let $2 \leq j \leq r$ and assume that indices $k_{1}, \ldots, k_{j-1}$ have been constructed such that

$$
\left[U^{k_{1}} \cup \cdots \cup U^{k_{j-1}}\right] \cap\left[\cup_{k \in\{1, \ldots, r\} \backslash\left\{k_{1}, \ldots, k_{j-2}\right\}} R^{i}\left(\varphi^{k}\right)\right]=\emptyset ;
$$

applying now property (ii) of Definition 4.1 with $J=\{1, \ldots, r\} \backslash\left\{k_{1}, \ldots, k_{j-1}\right\}$ we get $k_{j} \in J$ such that

$$
\left[U^{k_{1}} \cup \cdots \cup U^{k_{j}}\right] \cap\left[\cup_{k \in\{1, \ldots, r\} \backslash\left\{k_{1}, \ldots, k_{j-1}\right\}} R^{i}\left(\varphi^{k}\right)\right]=\emptyset ;
$$

Repeating the procedure $r$ times yields a permutation $\left(k_{1}, \ldots, k_{r}\right)$ with the desired properties.

$(2) \Rightarrow(1)$ : We check that $\left(U^{k}, \varphi^{k}\right)$ is a cycle in $\mathcal{E}$, and it suffices to show that (ii) of Definition 4.1 is fulfilled. Thus, let $i \in N$ and let $J$ be a nonempty subset of $\{1, \ldots, r\}$. Let $j^{0}$ be such that $J \subseteq\left\{k_{j^{0}}, \ldots, k_{r}\right\}$ where $\left(k_{1}, \ldots, k_{r}\right)$ is the permutation defined in (ii). Then each set $R^{i}\left(\varphi^{j}\right) \cap U^{k^{0}}$ for $j \geq j^{0}$, so that $U^{k_{j} 0}$ does not intersect any of the sets $R^{i}\left(\varphi^{j}\right)$, for $j \in J$, and we have shown that (ii) of Definition 4.1 is satisfied.

For proving equivalence of stability and acyclicity, we need the following lemma which is a slight extension of a classical topological result about normal spaces. Here and in the sequel, $\bar{W}$ denotes the closure of the set $W$.

Lemma 4.3 Let $\left(U_{i}\right)_{i=1, \ldots, p}$ be a finite collection of open sets in a normal topological space $E$. Then there are open sets $W_{i}(i=1, \ldots, n)$ with the following properties:

(i) $\bar{W}_{i} \subset U_{i}$ for $i=1, \ldots, p$,

(ii) $\cup_{i \in J} U_{i}=E \Rightarrow \cup_{i \in J} W_{i}=E$ for all subsets $J$ of $\{1, \ldots, p\}$.

Proof: Let $\mathcal{J} \subset \mathcal{P}(\{1, \ldots, p\})$ be the set of of subsets of indices such that $\cup_{i \in J} U_{i}=E$. For any $h \in\{1, \ldots, p\}$ let $\mathcal{J}_{h}=\{J \in \mathcal{J} \mid h \in J\}$. Without loss of generality we assume that $\mathcal{J}_{h} \neq \emptyset$ for all $h$. Then the sets $U_{h}^{c}$ and $\cap_{j \in J, j \neq h} U_{j}^{c}$ are disjoint for each $J \in \mathcal{J}_{h}$, so that

$$
U_{h}^{c} \cap\left[\cup_{J \in \mathcal{J}_{h}} \cap_{j \in J, j \neq h} U_{j}^{c}\right]=\emptyset .
$$

By normality of $E$, there are disjoint open sets $V$ and $W_{h}$ such that $U_{h}^{c} \subset V$ and $\left[\cup_{J \in \mathcal{J}_{h}} \cap_{j \in J, j \neq h} U_{j}^{c}\right] \subset W_{h}$. Clearly $\bar{W}_{h} \subset V^{c} \subset U_{h}$, and for each $J \in \mathcal{J}_{h}, W_{h}$ contains $\cup_{j \in J, j \neq h} U_{j}^{c}$, so that $\left(W_{h},\left(U_{j}\right)_{j \in J, j \neq h}\right)$ is a covering of $E$. Replacing the family $\left(U_{1}, \ldots, U_{p}\right)$ by $\left(W_{h},\left(U_{j}\right)_{j \neq h}\right)$ and repeating the procedure taking successively $h=1,2, \cdots, p$, we eventually get a family $\left(W_{1}, \ldots, W_{p}\right)$ with the desired properties. 
A family $\left(W_{i}\right)_{i=1}^{p}$ with the properties stated in Lemma 4.3 is called a refinement of $\left(U_{i}\right)_{i=1}^{p}$.

Now we are ready for the main result of this section. As explained in the beginning of the section, the technique of proof consists basically in showing that the cycle conditions posed on each individual are exactly those that allow for the construction of a particular assignment of preferences to individuals such that all alternatives become dominated.

Theorem 4.4 An interaction presheaf $\mathcal{E}: \mathcal{G}_{0} \rightarrow \mathcal{P}\left(\mathcal{F}_{0}(N, A)\right)$ is stable if and only if it is acyclic.

Proof. Assume that $\mathcal{E}$ is not stable. Then $\operatorname{Stl}(\mathcal{E}, u)$ is empty for some continuous profile $u=\left(u_{1}, \ldots, u_{n}\right)$, that is for any $a \in A$ there is $U^{a} \in \mathcal{G}$ and $\varphi^{a} \in \mathcal{E}\left[U^{a}\right]$ such that such that $\varphi^{a}(S) \subseteq P\left(a, S, u_{i}\right)$ for all $S \in \mathcal{P}_{0}(N)$, or expressed otherwise, $u_{i}(a)<\min \left\{u_{i}(b) \mid b \in R^{i}\left(\varphi^{a}\right)\right\}$ for all $i \in N$ such that $R^{i}\left(\varphi^{a}\right) \neq \emptyset$. Since the $u_{i}$ are continuous, there exists an open neighbourhood $W^{a}$ of a such that $\sup \left\{u_{i}(c) \mid c \in W^{a}\right\}<\min \left\{u_{i}(b) \mid b \in R^{i}\left(\varphi^{a}\right)\right\}$ for all $i \in N$ such that $R^{i}\left(\varphi^{a}\right) \neq \emptyset$. Moreover, by the presheaf property, $\varphi^{a} \in \mathcal{E}\left[U^{a} \cap W^{a}\right]$.

Since $A$ is compact, there exist $a_{1}, \ldots, a_{r}$ such that the family $\left(U^{a_{1}} \cap W^{a_{1}}, \ldots\right.$, $\left.U^{a_{r}} \cap W^{a_{r}}\right)$ is a covering of $A$. Put $V^{k}=U^{a_{k}} \cap W^{a_{k}}, \varphi^{k}=\varphi^{a_{k}}, k=1, \ldots, r$. We show that $\left(V^{k}, \varphi^{k}\right)_{k=1}^{r}$ is a cycle.

Clearly, $\cup_{k=1}^{r} V^{k}=A$; to check (ii) in Definition 4.1, let $i \in N$ and $\emptyset \neq J \subset$ $\{1, \ldots, r\}$. Let $k \in J$ such that $\sup \left\{u_{i}(c) \mid c \in V^{k}\right\}=\min _{j \in J} \sup \left\{u_{i}(c) \mid c \in V^{j}\right\}$. We claim that $V^{k} \cap R^{i}\left(\varphi^{j}\right)=\emptyset$ for all $j \in J$. Indeed, this is trivially true if $R^{i}\left(\varphi^{j}\right)=\emptyset$. If $R^{i}\left(\varphi^{j}\right) \neq \emptyset$, let $a \in V^{k}$ and $b \in R^{i}\left(\varphi^{j}\right)$. We then have

$$
u_{i}(a) \leq \sup _{c \in V^{k}} u_{i}(c) \leq \sup _{c \in V^{j}} u_{i}(c)<u_{i}(b),
$$

which proves the claim and shows that $\left(V^{k}, \varphi^{k}\right)_{k=1}^{r}$ is indeed a cycle.

Conversely let $\left(U^{k}, \varphi^{k}\right)_{k=1}^{r}$ be a cycle in $\mathcal{E}$. We construct a profile $\left(u_{1}, \ldots, u_{n}\right)$ such that $\operatorname{Stl}(\mathcal{E}, u)$ is empty. By Lemma 4.2 there is a permutation $\left(k_{1}, \ldots, k_{r}\right)$ of $(1, \ldots, r)$ such that for any $j \in\{1, \ldots, r\}$,

$$
\left[U^{k_{1}} \cup \cdots \cup U^{k_{j}}\right] \cap\left[R^{i}\left(\varphi^{k_{j}}\right) \cup \cdots \cup R^{i}\left(\varphi^{k_{r}}\right)\right]=\emptyset,
$$

and by Lemma 4.3 there is a refinement $\left(W^{k}\right)_{k=1}^{r}$ of $\left(U^{k}\right)_{k=1}^{r}$ such that

$$
\left[\bar{W}^{k_{1}} \cup \cdots \cup \bar{W}^{k_{j}}\right] \cap\left[R^{i}\left(\varphi^{k_{j}}\right) \cup \cdots \cup R^{i}\left(\varphi^{k_{r}}\right)\right]=\emptyset
$$

for each $j \in\{1, \ldots, r\}$. We construct for each $i \in N$ a continuous function $u_{i}$ on $A$ such that

$$
\begin{array}{ll}
u_{i}(c) \leq h-1 & \text { for } h \in\{1, \ldots, r\} \text { and } c \in \bar{W}^{k_{h}} \\
u_{i}(b) \geq h & \text { for } b \in R^{i}\left(\varphi^{k_{h}}\right) .
\end{array}
$$


This may be done as follows: Since $A$ is a normal topological space, there is for each $h \in\{1, \ldots, r\}$ a continuous function $v^{h}: A \rightarrow[0,1]$ such that

$$
v^{h}(a)=\left\{\begin{array}{l}
0 \text { if } a \in \bar{W}^{k_{1}} \cup \cdots \cup \bar{W}^{k_{h}} \\
1 \text { if } a \in R^{i}\left(\varphi^{k_{h}}\right) \cup \cdots \cup R^{i}\left(\varphi^{k_{r}}\right) .
\end{array}\right.
$$

The function $u_{i}=\sum_{h=1}^{r} v^{h}$ has the desired properties.

Consider now the profile $\left(u_{1}, \ldots, u_{n}\right)$. If $a \in \bar{W}^{j}$ and $i \in N$, then there exists an index $h$ (depending on $i$ ) such that $k_{h}=j$. It follows that $u_{i}(a) \leq h$ and for any $b \in R^{i}\left(\varphi^{j}\right), u_{i}(b) \geq h+1$, therefore $R^{i}\left(\varphi^{j}\right) \subset P\left(a, u_{i}\right)$ for each $i$, and by the presheaf property we have $P\left(W^{k}, \cdot, u\right) \in \mathcal{E}\left[W^{k}\right]$, so that every $a \in W^{k}$ is dominated. Since $\left(W_{1}, \ldots, W_{n}\right)$ is a covering of $A$, the set of undominated alternatives at the profile $\left(u_{1}, \ldots, u_{n}\right)$ is empty.

Remark 4.5 Theorem 4.4 has been proved for the class of continuous preferences representable by continuous functions. It is easy to see, by a slight modification of the first part of the proof, that the same result holds for the larger class of continuous preferences.

The characterization of stable interaction presheaves given in Theorem 4.4 gives a purely combinatorial property of the power structure, which in principle may be verified without recourse to preference profiles and notions of domination. In order to exploit this fact in characterizing solvable game forms, we need to investigate the relation between equilibria of the game form and the settlements of its associated interaction forms and bundles. This is done in the following section.

\section{Game form solvability and stability of inter- action forms}

In this section, we connect the notion of stability and its characterization in the previous section to that of solvability of game forms, the link being the $\beta$ interaction presheaf of the game form. Let $G=\left(X_{1}, \cdots, X_{n}, A, g\right)$ be a game form such that the strategy spaces $X_{i}$ for $i=1, \ldots, n$ as well as the outcome space $A$ are compact Hausdorff spaces, and where $g$ is continuous and onto. In Section 3 , we introduced the associated $\beta$-interaction presheaf $\mathcal{E}_{\beta}^{G}$. We assume that an ACS $\mathcal{M} \subset \mathcal{P}_{0}(N)$ is given. We define the associated $\beta$-interaction bundle $\mathcal{I}_{\beta} \equiv \mathcal{I}_{\beta}^{G, \mathcal{M}}$ by

$$
\begin{aligned}
\mathcal{I}_{\beta}[a]=\left\{\varphi \in \mathcal{P}_{0}(N, A) \mid\right. & \forall x_{N} \in g^{-1}(a), \\
& \left.\exists S \in \mathcal{M}, y_{S} \in X_{S}: g\left(y_{S}, x_{S^{c}}\right) \in \varphi(S)\right\}
\end{aligned}
$$


and the corresponding interaction sheaf (2). Similarly we define the $\alpha$-interaction bundle $\mathcal{I}_{\alpha} \equiv \mathcal{I}_{\alpha}^{G, \mathcal{M}}$ and the corresponding interaction form $\mathcal{E}_{\alpha} \equiv \mathcal{E}_{\alpha}^{G, \mathcal{M}}$ given by (3). One has $\varphi \in \mathcal{E}_{\beta}[U]$ if and only if $\varphi^{c} \notin \mathcal{E}_{\alpha}[U]$, where by definition $\varphi^{c}(S)=$ $\varphi(S)^{c}$ for all $S \in P_{0}(N)$. It is also clear that $\mathcal{E}_{\beta}=\left(\mathcal{I}_{\beta}\right)^{\diamond}$.

Remark 5.1 One advantage of our present model compared to that of Abdou and Keiding (2003) is that restrictions on active coalition structures as given in (1) reflect faithfully the shift of power from one ACS to another in the game form $G$. This is because we have:

$$
\mathcal{E}_{\beta}^{G, \mathcal{M}}=\left.\left(\mathcal{E}_{\beta}^{G, \mathcal{P}_{0}(M)}\right)\right|_{\mathcal{M}}
$$

where the object on the right-hand side is the projection of $\mathcal{E}_{\beta}^{G, \mathcal{P}_{0}(M)}$ on $\mathcal{M}$. It follows that one needs only to know $\mathcal{E}_{\beta}^{G, \mathcal{P}_{0}(M)}$ in order to deduce $\mathcal{E}_{\beta}^{G, \mathcal{M}}$ for all active coalition structures $\mathcal{M}$.

The following result is straightforward but central for our characterization of solvable game forms. It shows that the concept of $\beta$-interaction bundle is to the $\mathcal{M}$-equilibrium of the game $\left(G, R_{N}\right)$ what the $\beta$-effectivity function is to the $\beta$-core of the latter game:

Proposition 5.2 For any $R_{N}$ the set of $\mathcal{M}$-equilibrium outcomes of $\left(G, R_{N}\right)$ is equal to $\operatorname{Stl}\left(\mathcal{I}_{\beta}, R_{N}\right)$.

Proof: Let $a \in A$ be an $\mathcal{M}$-equilibrium outcome of $\left(G, R_{N}\right)$. There exists an $\mathcal{M}$-equilibrium $x_{N} \in X$ of $\left(G, R_{N}\right)$ such that $g\left(x_{N}\right)=a$ and for all $S \in \mathcal{M}$ and $y_{S} \in X_{S}, g\left(y_{S}, x_{S^{c}}\right) \notin P\left(a, S, R_{N}\right)$, and consequently, the interaction array $P\left(a, \cdot, R_{N}\right)$ does not belong to $\mathcal{I}_{\beta}[a]$. If follows that $a$ is not dominated in $\mathcal{I}_{\beta}[a]$ at $R_{N}$, or equivalently $a \in \operatorname{Stl}\left(\mathcal{E}_{\beta}, R_{N}\right)$.

Conversely, if $a \in \operatorname{Stl}\left(\mathcal{I}_{\beta}, R_{N}\right)$, then the interaction array $P\left(a, \cdot, R_{N}\right)$ is not in $\mathcal{I}_{\beta}[a]$. But then there must be some strategy array $x_{N} \in X$ with $g\left(x_{N}\right)=a$ such that $g\left(y_{S}, x_{S^{c}}\right) \notin P\left(a, S, R_{N}\right)$ for all $S \in \mathcal{M}$ and all $y_{S} \in X_{S}$, showing that $x_{N}$ is an $\mathcal{M}$-equilibrium.

With the result of Proposition 5.2 we are getting close to our goal, since we have identified the settlement set of $\stackrel{\circ}{\mathcal{I}}_{\beta}$ at $R_{N}$ with the set of $\mathcal{M}$-equilibria of $\left(G, R_{N}\right)$. What remains is to relate $\stackrel{\circ}{\mathcal{I}}_{\beta}$ to the $\beta$-effectivity sheaf, a somewhat technical task which is performed in the remainder of this section.

In order to apply the main result of Section 4, we need to work with interaction presheafs or interaction bundles which are either closed- or open-valued. Since however the relevant presheaf has a particular structure, we need a closer look at the $\beta$-interaction presheaf as well as other, related, constructions. We recall that the set of closed (open) valued interaction arrays is denoted $\mathcal{F}_{0}(N, A)\left(\mathcal{G}_{0}(N, A)\right)$. 
We define the presheaves $\overline{\mathcal{E}}_{\beta}\left(\stackrel{\circ}{\mathcal{E}}_{\beta}\right), \overline{\mathcal{E}}_{\alpha}\left(\stackrel{\circ}{\mathcal{E}}_{\alpha}\right)$ by restricting for each $U \in \mathcal{G}_{0}$ to the interaction arrays which take only closed (open) sets as values. Similarly, we introduce the interaction bundles $\overline{\mathcal{I}}_{\beta}, \stackrel{\circ}{\mathcal{I}}_{\beta}, \overline{\mathcal{I}}_{\alpha}\left(\stackrel{\circ}{\mathcal{I}}_{\alpha}\right)$.

Proposition 5.3 For any continuous $R_{N}$, the set of $\mathcal{M}$-equilibrium outcomes of $\left(G, R_{N}\right)$ is equal to $\operatorname{Stl}\left(\stackrel{\circ}{\mathcal{I}}_{\beta}, R_{N}\right)$.

We shall make use of a topology on the set of interaction arrays: For any $\psi \in$ $\mathcal{P}_{0}(N, A)$, define the lower interval $I_{\psi}$ and the upper interval $J_{\psi}$ by :

$$
\begin{aligned}
& I_{\psi}=\left\{\varphi \in \mathcal{F}_{0}(N, A) \mid \varphi \leq \psi\right\} \\
& J_{\psi}=\left\{\varphi \in \mathcal{G}_{0}(N, A) \mid \psi \leq \varphi\right\}
\end{aligned}
$$

The collection $\left\{I_{\psi} \mid \psi \in \mathcal{G}_{0}(N, A)\right\}$ is a basis for a topology on $\mathcal{P}_{0}(N, A)$ which is called the upper topology. $\mathcal{F}_{0}(N, A)$ will be endowed with its topology as a subspace of $\mathcal{P}_{0}(N, A)$.

Lemma 5.4 For any $a \in A$ and any $U \in \mathcal{G}_{0}$ we have:

(i) $\mathcal{I}_{\alpha}(a)=\left\{\varphi \in \mathcal{P}_{0}(N, A) \mid I_{\varphi} \cap \overline{\mathcal{I}}_{\alpha}(a) \neq \emptyset\right\}$

(ii) $\mathcal{E}_{\alpha}[U]=\left\{\varphi \in \mathcal{P}_{0}(N, A) \mid I_{\varphi} \cap \overline{\mathcal{E}}_{\alpha}[U] \neq \emptyset\right\}$

(iii) $\mathcal{I}_{\beta}(a)=\left\{\varphi \in \mathcal{P}_{0}(N, A) \mid J_{\varphi} \subset \stackrel{\circ}{\mathcal{I}}_{\beta}(a)\right\}$,

(iv) $\mathcal{E}_{\beta}[U]=\left\{\varphi \in \mathcal{P}_{0}(N, A) \mid J_{\varphi} \subset \dot{\mathcal{E}}_{\beta}[U]\right\}$

Proof: If $I_{\varphi} \cap \overline{\mathcal{I}}_{\alpha}(a) \neq \emptyset$ then clearly $\varphi \in \mathcal{I}_{\alpha}(a)$. Conversely, assume that $\varphi \in \mathcal{I}_{\alpha}(a)$, then there exists $x_{N} \in X_{N}$ such that $g\left(x_{N}\right)=a$ and for all $S \in \mathcal{M}$, $g\left(x_{S^{c}}, X_{S}\right) \subset \varphi(S)$. Since the sets $g\left(x_{S^{c}}, X_{S}\right)$ for $S \in \mathcal{P}_{0}(N)$ are closed, the interaction array $\psi$ defined by $\psi(S):=g\left(x_{S^{c}}, X_{S}\right),\left(S \in \mathcal{P}_{0}(N)\right)$ belongs to $\overline{\mathcal{I}}_{\alpha}(a)$ and $\psi \leq \varphi$. This proves assertion (i). The verification of the other assertions is left to the reader.

Lemma 5.5 The correspondence $\overline{\mathcal{I}}_{\alpha}$ from $A$ to $\mathcal{F}_{0}(N, A)$ has closed graph in $A \times \mathcal{F}_{0}(N, A)$.

Proof: Let $(a, \varphi) \in A \times \mathcal{F}_{0}(N, A)$, let $\mathcal{V}_{a}$ be the set of open neighbourhoods of $a$, let $\mathcal{V}_{\varphi}$ be the family $\mathcal{V}_{\varphi}:=\left(V: V \in \mathcal{G}_{0}(N, A), \varphi \leq V\right)$ and let $\mathcal{V}=\mathcal{V}_{a} \times \mathcal{V}_{\varphi}$. Assume that $(a, \varphi)$ belongs to the closure of the graph of $\overline{\mathcal{I}}_{\alpha}$, that is for any $V \equiv\left(V_{1}, V_{2}\right) \in \mathcal{V}$ there exist $b^{V} \in V_{1}$ and $\psi^{V} \in I_{V_{2}} \cap \overline{\mathcal{I}}_{\alpha}\left(b^{V}\right)$. By the definition of $\overline{\mathcal{I}}_{\alpha}$, there exists $x_{N}^{V} \in X_{N}$ such that $g\left(x_{N}^{V}\right)=b^{V}$ and for all $S \in \mathcal{M}, g\left(x_{S^{c}}^{V}, X_{S}\right) \subset$ $\psi^{V}(S) \subset V_{2}(S)$.

The set $\mathcal{V}$ ordered by componentwise inclusion is a directed set. Since $X$ is compact, the net $\left(x_{N}^{V}\right)_{V \in \mathcal{V}}$ admits a convergent subnet, say $\left(x_{N}^{V_{t}}\right)_{t \in T}$, where $(T, \geq)$ 
is a directed set. Let $x_{N}$ be its limit; by continuity of $g, g\left(x_{N}\right)=a$. We claim that for all $S \in \mathcal{M}, g\left(x_{S^{c}}, X_{S}\right) \subset \varphi(S)$. Indeed, for any $t \in T$, continuity of $g$ implies that $g\left(x_{S^{c}}, X_{S}\right) \subset \overline{V_{2, t}(S)}$. Let $V \in \mathcal{V}_{\varphi}$. Since $A$ is normal and due to the subnet property, there exists some $t \in T$ such that $\varphi(S) \subset \overline{V_{2, t}(S)} \subset V_{2}(S)$ for all $S \in \mathcal{S}$. Therefore $g\left(x_{S^{c}}, X_{S}\right) \subset V_{2}(S)$. Since the last inclusion is true for all $V_{2} \in \mathcal{V}_{\varphi}$, again by normality it follows that $g\left(x_{S^{c}}, X_{S}\right) \subset \varphi(S)$. We conclude that $\varphi \in \overline{\mathcal{I}}_{\alpha}(a)$.

Lemma $5.6 \stackrel{\circ}{\mathcal{I}}_{\beta}$ is regular: $\left(\stackrel{\circ}{\mathcal{E}}_{\beta}\right)^{\bullet}=\stackrel{\circ}{\mathcal{I}}_{\beta}$.

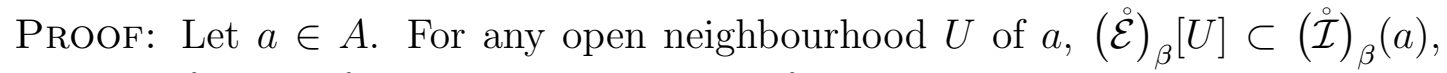
so that $\left(\dot{\mathcal{E}}_{\beta}\right)^{\bullet} \subset \stackrel{\circ}{\mathcal{I}}_{\beta}$. Conversely, if $\varphi \in\left(\stackrel{\mathcal{I}}{)_{\beta}}(a)\right.$ then $\varphi^{c} \notin \overline{\mathcal{I}}_{\alpha}(a)$, where $\varphi^{c}$ is the interaction array defined by $\varphi^{c}(S)=\varphi(S)^{c}$, all $S$. It follows from Lemma 5.5 that there exist $U \in \mathcal{G}_{0}, W \in \mathcal{G}_{0}(N, A)$ such that $a \in U, \varphi^{c} \in I_{W}$ and for all $b \in U, \overline{\mathcal{I}}_{\alpha}(b) \cap I_{W}=\emptyset$; in view of Lemma $5.4(\mathrm{i}), W \notin \dot{\mathcal{I}}_{\alpha}(b)$ or equivalently $W^{c} \in \overline{\mathcal{I}}_{\beta}(b)$. Since $W^{c}(S) \subset \varphi(S)$ for all $S \in \mathcal{P}_{0}(N)$, we have $\varphi \in \stackrel{\circ}{\mathcal{I}}_{\beta}(b)$. Since this is true for any $b \in U$, we get that $\varphi \in\left(\stackrel{\circ}{\mathcal{I}}_{\beta}\right)^{\diamond}(U) \equiv \stackrel{\circ}{\mathcal{E}}_{\beta}[U]$, and since $a \in U$ we have $\varphi \in(\stackrel{\mathcal{I}}{\diamond})_{\beta}^{\diamond}[U]$.

Example 5.7 Let $A:=X_{1} \times X_{2}$, let $\pi: X_{1} \times X_{2} \rightarrow X_{1} \times X_{2}$ be the identical map on $A$, and let $\pi_{i}$ for $i=1,2$ be the projections. Let $\mathcal{M}=\{\{1\}\}$. For any $\left(x_{1}, x_{2}\right) \in A, U \in \mathcal{G}_{0}$, let

$$
\begin{aligned}
& I_{\beta}\left(x_{1}, x_{2}\right)=\left\{B \subset A \mid x_{2} \in \pi_{2}(B)\right\}, \\
& E_{\beta}[U]=\left\{B \subset A \mid \pi_{2}(U) \subset \pi_{2}(B)\right\},
\end{aligned}
$$

then we have:

$$
\begin{aligned}
\mathcal{I}_{\beta}\left(x_{1}, x_{2}\right) & =\left\{\varphi \mid \varphi(1) \in I_{\beta}\left(x_{1}, x_{2}\right)\right\}, \\
\mathcal{E}_{\beta}(U) & =\left\{\varphi \mid \varphi(1) \in E_{\beta}[U]\right\} .
\end{aligned}
$$

We remark that the set $\left\{\left(y_{1}, x_{2}\right)\right\} \in I_{\beta}\left(x_{1}, x_{2}\right)$, but unless $x_{2}$ is isolated, there is no $U \in \mathcal{U}_{\left.\left(x_{1}, x_{2}\right)\right)}$ such that $\pi_{2}(U)=\left\{x_{2}\right\}$. Therefore, unless $X_{2}$ is finite, we must have that $\mathcal{E}_{\beta}^{\bullet} \neq \mathcal{I}_{\beta}$. It follows that unless $X_{2}$ is finite, $\mathcal{I}_{\beta}$ and $\overline{\mathcal{I}}_{\beta}$ are not regular. Moreover, if $R: X_{2} \rightarrow X_{1}$ is a map, then the graph of $R$, $\operatorname{Graph}(R)$, is an element of $E_{\beta}[A]$, but unless $R$ is continuous, there is no closed $B \in E_{\beta}[A]$ such that $B \subset \operatorname{Graph}(R)$. It follows that there is no analog of Lemma 5.4(i) for $\mathcal{I}_{\beta}$.

Lemma 5.8 For any $a \in A$ and any $U \in \mathcal{G}_{0}$ we have:

(i) $\stackrel{\circ}{\mathcal{I}}_{\beta}(a)=\left\{V \in \mathcal{G}_{0}(N, A) \mid I_{V} \cap \overline{\mathcal{I}}_{\beta}(a) \neq \emptyset\right\}$.

(ii) $\dot{\mathcal{E}}_{\beta}[U]=\left\{V \in \mathcal{G}_{0}(N, A) \mid I_{V} \cap \overline{\mathcal{E}}_{\beta}[U] \neq \emptyset\right\}$, 
Proof: By the definitions, if $V \in \mathcal{G}_{0}(N, A)$ and $I_{V} \cap \overline{\mathcal{I}}_{\beta}(a) \neq \emptyset$, then $V \in \dot{\mathcal{E}}_{\beta}(a)$. Conversely, if $V \in \stackrel{\circ}{\mathcal{I}}_{\beta}(a)$, then $V^{c} \notin \overline{\mathcal{I}}_{\alpha}(a)$, so that by Lemma 5.5 there exists $W \in \mathcal{G}_{0}(N, A)$ such that $V^{c} \in I_{W}$ and $I_{W} \cap \overline{\mathcal{I}}_{\alpha}(a)=\emptyset$. In view of Lemma 5.4(i), $W \notin \dot{\mathcal{I}}_{\alpha}(a)$, or equivalently $W^{c} \in \overline{\mathcal{I}}_{\beta}(a)$. Since $W^{c} \in I_{V}$, we have $W^{c} \in$ $I_{W} \cap \overline{\mathcal{I}}_{\beta}(a)$. This proves (i). The proof of the other assertion is left to the reader.

Lemma 5.9 For each continuous profile $R_{N}$ we have:

$$
\operatorname{Stl}\left(\mathcal{I}_{\beta}, R_{N}\right)=\operatorname{Stl}\left(\stackrel{\circ}{\mathcal{I}}_{\beta}, R_{N}\right)=\operatorname{Stl}\left(\stackrel{\circ}{\mathcal{E}}_{\beta}, R_{N}\right)=\operatorname{Stl}\left(\overline{\mathcal{I}}_{\beta}, R_{N}\right)=\operatorname{Stl}\left(\overline{\mathcal{E}}_{\beta}, R_{N}\right)
$$

and this set equals the set of equilibrium outcomes of $G$ at $R_{N}$.

Proof: Let $R_{N}$ be a continuous profile. Put $V(S)=P\left(a, S, R_{N}\right)\left(S \in P_{0}(N)\right)$. Clearly $V$ has open values. The first equality follows by Proposition 5.3. The second equality follows from regularity of $\dot{\mathcal{I}}_{\beta}$ (Lemma 5.6). In view of Lemma 5.8(i), $\operatorname{Stl}\left(\stackrel{\mathcal{I}}{\beta}_{\beta}, R_{N}\right)=\operatorname{Stl}\left(\overline{\mathcal{I}}_{\beta}, R_{N}\right)$. From Lemma 5.8(ii), we get that $\operatorname{Stl}\left(\dot{\mathcal{E}}_{\beta}, R_{N}\right)=$ $\operatorname{Stl}\left(\overline{\mathcal{E}}_{\beta}, R_{N}\right)$.

Now we have all the ingredients for the main result of this section, and indeed of the paper, which provides a combinatorial necessary and sufficient condition for solvability of a game form.

Theorem 5.10 $G$ is $\mathcal{M}$-solvable if and only if $\overline{\mathcal{E}}_{\beta}\left(\dot{\mathcal{E}}_{\beta}\right)$ is acyclic.

Proof. Applying Theorem 4.4 to $\overline{\mathcal{E}}_{\beta}$, one has that $\mathcal{M}$-solvability of $G$ is equivalent to acyclicity of the interaction sheaf $\overline{\mathcal{E}}_{\beta}$. Moreover, by Lemma 5.8(ii) any cycle of $\dot{\mathcal{E}}_{\beta}$ gives rise to a cycle of $\overline{\mathcal{E}}_{\beta}$.

Conversely, given a cycle $\left(C^{k}, \varphi^{k}\right)_{k=1}^{r}$ of $\overline{\mathcal{E}}_{\beta}$, applying Lemma 4.3 one may replace the open covering $\left(C^{k}\right)_{k=1}^{r}$ of $A$ by an open covering $\left(U^{k}\right)_{k=1}^{r}$ such that $\bar{U}^{k} \subset C^{k}$ for $k=1, \ldots, r$. The array $\left(\bar{U}^{k}, \varphi^{k}\right)_{k=1}^{r}$ still verifies the combinatorial properties of cycles. By a further application of Lemma 4.3 to the family $\left(\left(\bar{U}^{k}\right)^{c},\left(\varphi^{k}(S)\right)^{c}, k=1, \cdots, r, S \in P_{0}(N)\right)$ of open sets, one can replace $\left(\bar{U}^{k}, \varphi^{k}\right)$ by $\left(W^{k}, \psi^{k}\right)$, taking open values such that $\bar{U}^{k} \subset W^{k}$ and $\varphi^{k}(S) \subset \psi^{k}(S)$ for all $S \in \mathcal{P}_{0}(N), k=1 \cdots, r$, thus getting a family $\left(U^{k}, \psi^{k}\right)_{k=1}^{r}$ which satifies conditions (i) and (ii) in the definition of cycles. Since $U^{k} \subset C^{k}$, one has $\psi^{k} \in \stackrel{\mathcal{E}}{\beta}_{\beta}\left[C^{k}\right] \subset \stackrel{\circ}{\mathcal{E}}_{\beta}\left[U^{k}\right]$. Thus, $\left(U^{k}, \psi^{k}\right)_{k=1}^{r}$ is a cycle in $\dot{\mathcal{E}}_{\beta}$.

\section{Interaction sheaves over convex domains}

In the present section, we consider a special case which however turns up in many applications, namely that where the domain $A$ is a convex and compact 
subset of some Euclidean space $\mathbb{R}^{d}$. Let $\mathcal{C}$ denote the set of all convex and closed subsets of $A$ and $\mathcal{C}_{0}(A, N)$ the set of all interaction arrays $\varphi$ such that $\varphi(S) \in \mathcal{C}$ for all $S \in \mathcal{P}_{0}(N)$. Working with convex domains, it seems natural to restrict preferences to all $R \in Q(A)$ which are convex in the sense that for each $a \in A$, the set $P(a, R)$ is convex. Denoting this subset of $Q(A)$ by $Q_{\mathcal{C}}(A)$, we say that an interaction presheaf is $c$-stable if $\operatorname{Stl}\left(\mathcal{E}, R_{N}\right) \neq \emptyset$ for each $R_{N} \in Q_{\mathcal{C}}(A)^{N}$. Adding a linear structure of the outcome space means that in some cases, the properties of acyclicity and consequently of $\mathcal{M}$-solvability may take another form due to the restriction on the set of admissible preferences.

We then have to revise the results in Section 4 so as to take the convexity of domain and preferences into consideration. For this, we must modify the definition of a cycle given in Definition 4.1. We use the notation $\operatorname{co}(B)$ for the convex hull of $B \subset A$.

Definition 6.1 Let $\mathcal{E}$ be an interaction sheaf on $(N, A)$, A convex cycle in $\mathcal{E}$ is a family $\left(U^{k}, \varphi^{k}\right)_{k=1}^{r}$ with $U^{k} \in \mathcal{G}_{0}$ and $\varphi^{k} \in \mathcal{E}\left[U^{k}\right]$, each $k$, such that

(i) $\cup_{k=1}^{n} U^{k}=A$,

(ii) if $i \in N$ and $\emptyset \neq J \subset\{1, \ldots, r\}$ then there exists $k \in J$ such that $U^{k} \cap \operatorname{co}\left(\cup_{j \in J} R^{i}\left(\varphi^{j}\right)\right)=\emptyset$.

Lemma 4.2 is still valid in this context provided that its condition (2) is suitably reformulated.

Lemma 6.2 Let $\mathcal{E}$ be an interaction sheaf. Let $\mathcal{Z}=\left(W^{k}, \varphi^{k}\right)_{k=1}^{r}$ be a family with $W^{k} \in \mathcal{G}_{0}, \varphi^{k} \in \mathcal{E}\left[W^{k}\right], k=1, \ldots, r$, and $\cup_{k=1}^{n} W^{k}=A$. Then the following are equivalent:

(1) $\mathcal{Z}$ is a convex cycle of $\mathcal{E}$,

(2) For each $i \in N$ there is a permutation $\left(k_{1}, \ldots, k_{r}\right)$ of $(1, \ldots, r)$ such that for any $j \in\{1, \ldots, r\}$,

$$
\left[W^{k_{1}} \cup \cdots \cup W^{k_{j}}\right] \cap \operatorname{co}\left(\left[R^{i}\left(\varphi^{k_{j}}\right) \cup \cdots \cup R^{i}\left(\varphi^{k_{r}}\right)\right]\right)=\emptyset .
$$

Let $\mathcal{C}_{0}(A, N)$ be the set of all interaction arrays that are closed-and-convex valued. We define $\mathcal{E}_{\mathcal{C}}[U]=\mathcal{E}[U] \cap \mathcal{C}_{0}(A, N)$.

Lemma $6.3 \mathcal{E}$ is acyclic if and only if $\mathcal{E}_{\mathcal{C}}$ is acyclic. $\mathcal{E}$ is stable on $Q_{\mathcal{C}}$ if and only if $\mathcal{E}_{\mathcal{C}}$ is stable.

Proof: Clearly any cycle in $\mathcal{E}_{\mathcal{C}}$ is a cycle in $\mathcal{E}$. Conversely, let $\left(W^{k}, \varphi^{k}\right)_{k=1}^{r}$ be a cycle in $\mathcal{E}$. If for each $k$, we replace $\varphi^{k}$ by $\varphi^{\prime k}$, where $\varphi^{\prime k}(S)=\operatorname{co}\left(\varphi^{k}(S)\right)$ for all $S \in \mathcal{P}_{0}(N)$, we obtain a cycle $\left(W^{k}, \varphi^{\prime k}\right)_{k=1}^{r}$ in $\mathcal{E}_{\mathcal{C}}$. Therefore $\mathcal{E}$ is acyclic if and only if $\mathcal{E}_{\mathcal{C}}$ is acyclic. We have a similar argument for stability.

Before proving the counterpart of Theorem 4.4 in the context of convex cycles, we need the following: 
Lemma 6.4 Let $C_{1} \subset \cdots \subset C_{p}$ be an increasing sequence of compact and convex sets of $\mathbb{R}^{d}$ such that $0 \in \stackrel{\circ}{C}_{1}$ and $C_{k} \subset \stackrel{\circ}{C}_{k+1}$ for $k=1, \ldots, p-1$. Then there exists a continuous quasiconvex function $v$ such that

$$
v(x) \leq k \Longleftrightarrow x \in C_{k}, k=1, \ldots, p .
$$

Proof: For any convex set containing 0 , denote by $J_{C}(x)=\inf \{\lambda>0 \mid x \in \lambda C\}$ $(=+\infty$ if the latter set is empty) and let $\partial C$ denote the boundary of $C$. Define $v$ as follows:

$$
v(x)=\left\{\begin{array}{ll}
J_{C_{1}}(x) & \text { if } x \in C_{1}, \\
k+\frac{J_{C_{k}}(x)-1}{J_{C_{k}}(x)-J_{C_{k+1}}(x)} & \text { if } x \in C_{k+1} \backslash C_{k} \\
p-1+J_{C_{p}}(x) & \text { if } x \in \mathbb{R}^{d} \backslash C_{p}
\end{array}(k=1, \cdots, p-1)\right.
$$

Then $v$ is continuous on any point $x \in \mathbb{R}^{d} \backslash \cup_{k=1}^{p} \partial C_{k}$ since the functions $J_{C_{k}}$ are continuous and $J_{C_{k}}(x)-J_{C_{k+1}}(x)>0$ for $x \neq 0$. Moreover if $x \in \partial C_{k}$ then $v(x)=k$ so that one can verify easily the continuity of $v$ at $x$.

Now we verify quasiconvexity of $v$ : let $x, y \in \mathbb{R}^{d}, \lambda \in[0,1]$, and let $z=$ $(1-\lambda) x+\lambda y$. Assume $v(x) \leq v(y)$. We distinguish 3 cases:

Case 1: $y \in C_{1}$. We have $x \in C_{1}$ so that by convexity of $J_{C_{1}}, v(z) \leq$ $\max \{v(x), v(y)\}$.

Case 2: $y \in C_{k+1} \backslash C_{k}$ where $1 \leq k \leq p-1$. Let $\alpha=v(y)-k$. If $z \in C_{k}$ then $v(z) \leq v(y)$. If $z \in C_{k+1} \backslash C_{k}$ then

$$
\begin{aligned}
(1-\alpha) J_{C_{k}} & (z)+\alpha J_{C_{k+1}}(z) \\
& \leq(1-\alpha)\left[(1-\lambda) J_{C_{k}}(x)+\lambda J_{C_{k}}(y)\right]+\alpha\left[(1-\lambda) J_{C_{k+1}}(x)+\lambda J_{C_{k+1}}(y)\right] \\
& =(1-\lambda)\left[(1-\alpha) J_{C_{k}}(x)+\alpha J_{C_{k+1}}(x)\right]+\lambda\left[(1-\alpha) J_{C_{k}}(y)+\alpha J_{C_{k+1}}(y)\right] \\
& \leq(1-\lambda)+\lambda=1 .
\end{aligned}
$$

Here the first inequality follows from convexity of $J_{C_{k}}$ and $J_{C_{k+1}}$ and the fact that $0 \leq \alpha \leq 1$. When $x \in C_{k}$, we have that $J_{C_{k}}(x) \leq 2$ and $J_{C_{k+1}}(x) \leq 1$, so that $(1-\alpha) J_{C_{k}}(x)+\alpha J_{C_{k+1}}(x) \leq 1$. When $x \in \mathcal{C}_{k+1} \backslash C_{k}$, then $v(x)-k \leq \alpha$, so that again $(1-\alpha) J_{C_{k}}(x)+\alpha J_{C_{k+1}}(x) \leq 1$. In both cases $v(y)-k=\alpha$, so that $(1-\alpha) J_{C_{k}}(y)+\alpha J_{C_{k+1}}(y)=1$. This justifies the second inequality. We conclude that $v(z)-k \leq \alpha$ or equivalently $v(z) \leq v(y)$.

Case 3: $y \in \mathbb{R}^{d} \backslash C_{p}$. If $z \in C_{p}$ then $v(z) \leq p \leq v(y)$. If $z \in \mathbb{R}^{d} \backslash C_{p}$ then either $x \in \mathbb{R}^{d} \backslash C_{p}$ and by convexity of $J_{C_{p}}$ we have $v(z) \leq v(y)$ or $x \in C_{p}$ then $J_{C_{p}}(x) \leq 1$ than again by convexity of $J_{C_{p}}$ we have $J_{C_{p}}(z) \leq \max 1, J_{C_{p}}(y)=J_{C_{p}}(y)$ so that $v(z) \leq v(y)$.

Now we have the ingredients for proving a counterpart of Theorem 4.4. Since the method of proof is the same, once we have established the necessary ingredients in the form of Lemma 6.2-6.4 above, we omit the details and present only an outline of the proof. 
Theorem 6.5 Let $\mathcal{E}$ be an interaction presheaf. Then $\mathcal{E}$ is stable on $Q_{\mathcal{C}}(A)$ if and only if $\mathcal{E}$ has no convex cycles.

Proof: (Outline) If $\mathcal{E}$ is not stable on $Q_{\mathcal{C}}(A)$, then existence of a convex cycle can be proved following the reasoning of the general case, with the only addition that the empty intersections of condition 2(ii) of Lemma 4.2 extend to the convex hull of the sets $\left.R^{i}\left(\varphi^{k_{j}}\right) \cup \cdots \cup R^{i}\left(\varphi^{k_{r}}\right)\right)$, so that condition 2(ii) of Lemma 6.2 is satisfied.

Conversely, if a convex cycle exists, then for every player $i$, let $\left(k_{1}, \ldots, k_{r}\right)$ the permutation given in (2) of Lemma 6.2 and let $B_{j}^{i}=\operatorname{co}\left(R^{i}\left(\varphi^{k_{j}}\right) \cup \cdots \cup R^{i}\left(\varphi^{k_{r}}\right)\right)$. Removing any empty $B_{k}^{i}$ from the list if necessary, we have that $B_{r}^{i} \subset \cdots \subset B_{1}^{i}$ is a decreasing family of compact and convex sets. Moreover the sets $B_{k}^{i}$ may be expanded is such a way that $B_{r}^{i}$ has nonempty interior, $B_{k}^{i} \subset \stackrel{\circ}{B} \stackrel{i}{k-1}>0$ for $k=2, \ldots, p$, and all intersection properties are kept. Then Lemma 6.4 may be applied to obtain a continuous quasiconvex function $v^{i}$ such that $v^{i}(x) \leq p^{i}+1-k$ if and only if $x \in B_{k}^{i}$. The profile $\left(u_{1}, \cdots, u_{n}\right)$, where $u^{i}=-v^{i}(x)(i=1, \cdots, n)$, will then have an empty settlement set.

\section{Concluding remarks}

In the previous sections, we have introduced the concept of an interaction sheaf and used it for the characterization of solvable game forms. This was done in a topological framework. Equivalence between acyclicity and stability is proved for the class of continuous preferences. In fact the Hausdorff assumption on the compact set $A$ provides a class of continuous preferences rich enough to separate closed sets by respecting some combinatorial property. The results are thus similar to those of the discrete framework (e.g. Abdou and Keiding (2003)). If the context requires restricted domains of preferences, the notion of acyclicity has to be modified in accordance to that domain (see Kolpin (1991) for the effectivity function case). As an interesting framework for this restriction we considered the case of convex domains and convex continuous preferences.

The interaction sheaf represents conflicts in an intrinsic way, since strategy sets are not explicitly described. The interpretation of an interaction sheaf adopted thoughout this paper is of the $\beta$-type. The power described is the upsetting power, the dual of which would be the stabilizing, or forcing power. In consistency with this interpretation, the interaction sheaf of a game form as presented here contains exactly the information needed to decide upon the question of solvability, and it cannot be excluded that future problems may need a further development of the concepts used, so that we might not yet have reached the final form of describing the power structure in a game form. However, the interaction sheaf seems to be suitable for many problems, of which we have only touched upon a few. Also, 
it should be observed that the construction may be applied not only to strategic game forms but also to conflict situations which are presented in a less simple form (indexed families of game forms, generalized game forms), pointing to a more basic role of the interaction sheaf for analyzing conflict situations. They may be either very simple, if they reflect the effectivity, where each coalition acts separately, or more complex, where individuals act jointly (Nash) and even more complex when all coalitions act jointly (strong Nash). But since all those specific forms can be extracted by projection from a unique form, the model allows for comparision of different contextual interactions, and it may be applied to an investigation of degrees of instability in cases where stability is not achieved. A closer study of these possibilities will however be a matter of future research.

\section{References}

Abdou, J., Keiding H., 1991. Effectivity Functions in Social Choice, Kluwer Academic Publishers, Dordrecht.

Abdou, J. and H.Keiding (2003), On necessary and sufficient conditions for solvability of game forms, Mathematical Social Sciences 46, 243 - 260.

Bredon, G. E. (1990), Sheaf Theory, Graduate texts in Mathematics 170, Springer Verlag.

Keiding, H., 1985. Necessary and sufficient conditions for stability of effectivity functions, International Journal of Game Theory, 93 - 101.

Kolpin, V. (1991), Mixed Effectivity and the essence of stability, Social Choice and Welfare $8,51-63$.

Moulin, H. and B.Peleg (1982), Cores of effectivity functions and implementation theory, Journal of Mathematical Economics 10, 115 - 162.

Nakamura, K. (1979), The vetoers in a simple game with ordinal preferences, International Journal of Game Theory 8, issue 1, 55-61.

Peleg, B. (1998), Effectivity functions, game forms, games, and rights, Social Choice and Welfare 15, $67-80$. 